\title{
Arbor
}

\section{Dos maneras de impulsar el arte: Peggy Guggenheim y Gertrude Vanderbilt}

\section{Carmen Rocamora García-Iglesias}

Arbor CLXVIII, 663 (Marzo 2001), 371-378 pp.

Recogemos en este artículo, la biografía de dos mujeres privilegiadas por la vida.

Peggy Guggenheim y GertrudeVanderbilt, tuvieron la posibilidad económica y social de impulsar a pintores de distintas procedencias y vanguardias.

Sin la primera, el Surrealismo hubiese sido imposible, y, su triunfo, su comprensión y su custodia, se habría esfumado, siendo considerados sus representantes como un grupo de excéntricos deseosos de escandalizar a un público que no entendía su concepto del Arte, y, solo se quedaba en los desenfrenos, las frases sin sentido, las orgías ó el snobismo de algunos de sus representantes.

La recopilación que hizo Peggy Guggenheim de esta vanguardia, hace comprender al estudioso del Arte que, por detrás de esas arrogancias individuales, había un credo común, un deseo de captar la comprensión del sueño, cuando la razón no está en vigilia, un automatismo psíquico ajeno al razonamiento $y$, una experimentación de los ámbitos hasta entonces desconocidos como el subconsciente, el azar, la locura ó los estados alucinatorios.

Peggy Guggenheim pudo haber empleado su posición económica en viajar, consumir ó tirar su dinero alegremente. No lo hizo así. Su posible excentricidad y sus escándalos, los canalizó por la vía del Arte, ayudando a sus amigos, abriendo Galerías increíbles como la del Soho de Nueva York, en la que: «...Los cuadros estaban montados sobre bates de béisbol, $y$, las luces se encendían y apagaban componiendo triángulos colgados de cuerdas como si flotaran en el espacio"...

$Y$ finalmente su auténtico amor por el Arte le llevó a comprar el Pallazo Vernier dei Leoni en Venecia, donde, ya sin excentricidades, el es- 
pectador puede contemplar y comprender la vanguardia que ella tanto amó.

Desde otro punto de vista, Gertrude Vanderbilt, utilizó su puesto en la sociedad para impulsar, no solo a sus amigos sino a aquellos pintores, que, principiantes, carecían de nombre, pero tenían talento, carácter y originalidad. Ella supo ver esas cualidades en los artistas que impulsó, y generó una situación nueva, poco seguida por ciertos Galeristas actuales que apuestan por la seguridad, el nombre o la firma de los autores, sin mirar su auténtico valor artístico.

Vanderbilt fue una gran mujer. Luchó contra los contratiempos de la vida, la incomprensión de sus contemporáneos y el concepto arcaico de los Museos de albergar solo a aquellos valores conocidos mundialmente.

A ella deben su popularidad muchos artistas, como Calder, Pollock, los representantes de la Escuela de Nueva York, al igual que vanguardias difíciles de comprender como el Constructivismo, el Minimal Art, o tantas otras...

El Museo Whitney de Nueva York, su gran creación, fue y será el trampolín del triunfo de infinitos pintores.

Sin su perseverancia, sin su existencia, sin su lucha constante y sin su ánimo inflexible, muchos de estos artistas hubiesen sido desconocidos y me atrevo a decir que alguna vanguardia, hubiese pasado fugazmente, si Gertrude Vanderbilt no hubiese comprado ciertos cuadros para colgarlos en su Museo.

La independencia de criterio, el ser plenamente consecuente con los propios actos y criterios, hacen que hoy, personas como yo, sintamos una enorme admiración por estas mujeres, que apostaron por el Arte, como móvil de sus vidas y como realización de sus personalidades fuertes, enérgicas y triunfalistas.

\section{Peggy Guggenheim}

Si tuviese que elegir una mujer como la mayor impulsora del Arte del s. XX, no dudaría en escoger a Peggy Guggenheim, que, entre excentricidades, caprichos, aventuras estrafalarias y una moral dudosa para su tiempo, fue la gran incitadora de ese Grupo fantástico, que, basándose en el Dadaismo, la Pintura Metafísica y las Teorías del psico análisis de Freud, fue capaz de llevar al lienzo, la interpretación de lo onírico, el sig- 
Dos maneras de impulsar el arte: Peggy Guggenheim y...

nificado de los sueños, el automatismo como vía de transmisión del subconsciente, en una palabra, la gran vanguardia $\mathrm{Su}$ rrealista.

Coraje y visión, generosidad y humildad, dinero y tiempo, un fuerte sentido de la responsabilidad histórica, unidos a una devoradora sensación estética, son los rasgos del carácter de esta mujer, que, no solo fue la coleccionista que reunía obras de arte por pura complacencia ó filantropía, sino alguien que sintió una cierta responsabilidad hacia la pintura y los artistas, y que poseyó los medios y la voluntad de actuar en pro de ese sentimiento.

La primera Galería, titulada: «Guggenheim Joven», fue abierta por ella en Londres, con el apo-

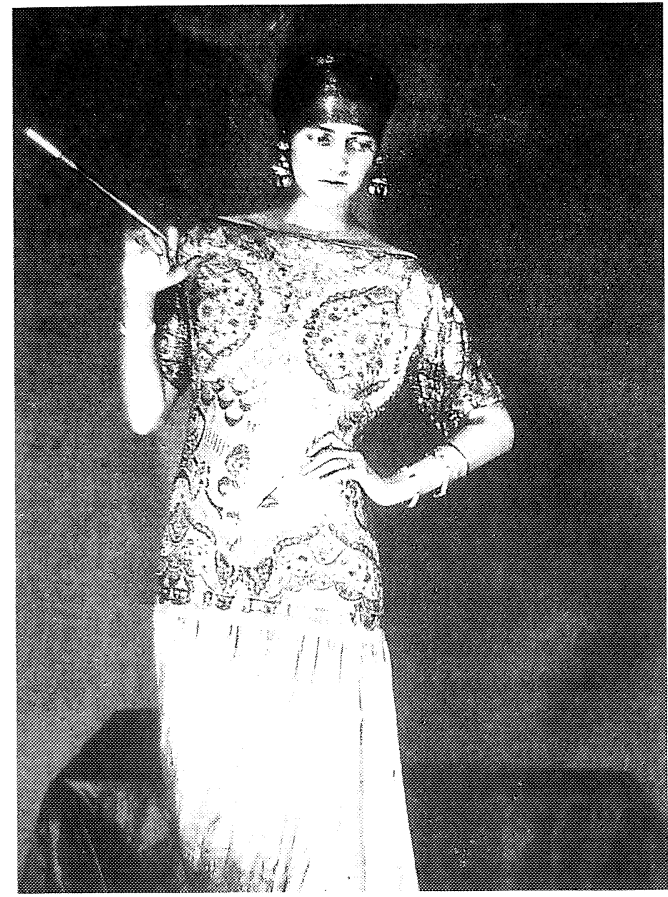

PEGGY GUGGENHEIM. «Retrato». yo y el consejo artístico de su amigo Duchamp. En ella, expuso Kandinsky (primer pintor abstracto del mundo), así como Yves Tanguy, uno de sus muchos amigos Surrealistas.

En 1939, su imparable carácter le llevó a la idea de abrir un «Museo de Arte Moderno» en Londres, y su deseo de rodearse de la gente más valiosa, le hizo elegir a Herbert Read, como Director de este deseado Museo. La impulsora y el Director, diseñaron una lista ideal de obras de arte para llevar a cabo la Primera Exposición, incluso llegaron a encontrar un edificio donde ubicarlo.

Pero, la II Guerra Mundial, puso fin a esta idea tan deseada por Peggy Guggenheim. Durante aquel invierno se trasladó a París, y allí se dedicó ampliar su Colección, comprando, bajo la supervisión de Duchamp y Van Doesburg «un cuadro cada día». Pero mientras ella alquilaba un lugar en la Place Vendome como emplazamiento de su nueva Galería, los alemanes se fueron acercando a París y, Peggy vio frustrado de nuevo su deseo de impulsar el Arte.

Durante el primer año de la ocupación nazi de París, la colección se puso a salvo en el Museo de Grenoble, aunque sin ser expuesto al público, por miedo a las represalias del régimen de Vichy. 


\section{Carmen Rocamora García-Iglesias}

374

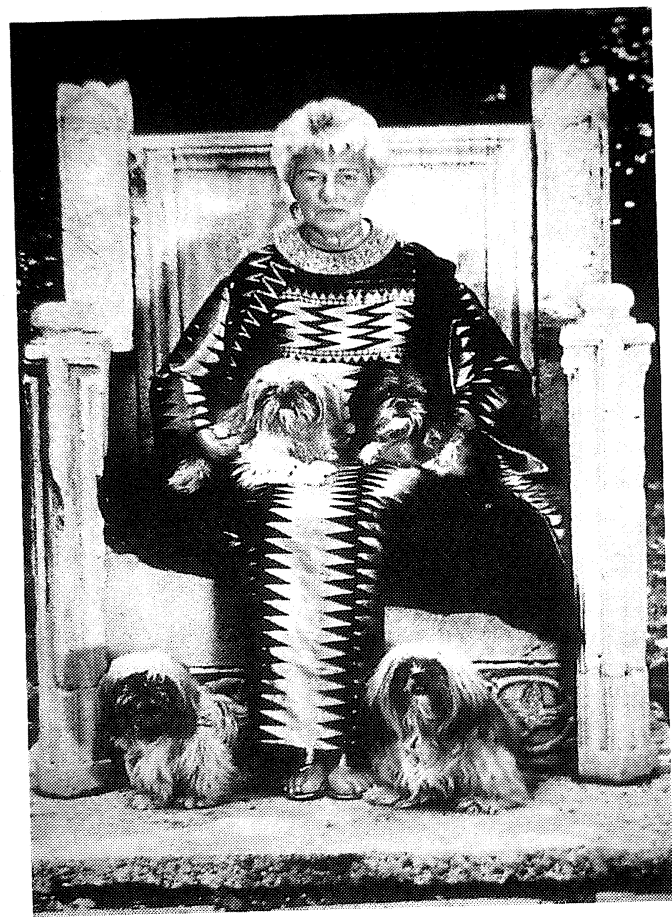

PEGGY GUGGENHEIM. «Retrato sobre asiento de mármol».
Por fin, en la primavera de 1941, Peggy, trasladó sus cuadros a Nueva York, como la mayor parte de los artistas europeos, que, huyendo de la Guerra, se instalaron en la ciudad de los rascacielos, por lo que París, dejó de ser el centro cultural y artístico del mundo, para trasladar esta supremacía a Nueva York.

Bajo el consejo de su entonces marido, Max Ernst, y del poeta André Breton, Peggy inauguró la más provocativa, excéntrica y estrafalaria Galería del Soho Neoyorkino, titulándola «The Art of this Century».

En ella, el Surrealismo, representado por Duchamp, Breton y Max Ernst pudo ser visto y entendido, aunque también se abrieron las puertas a pintores de otras tendencias como el Construtivista Piet Mondrian.

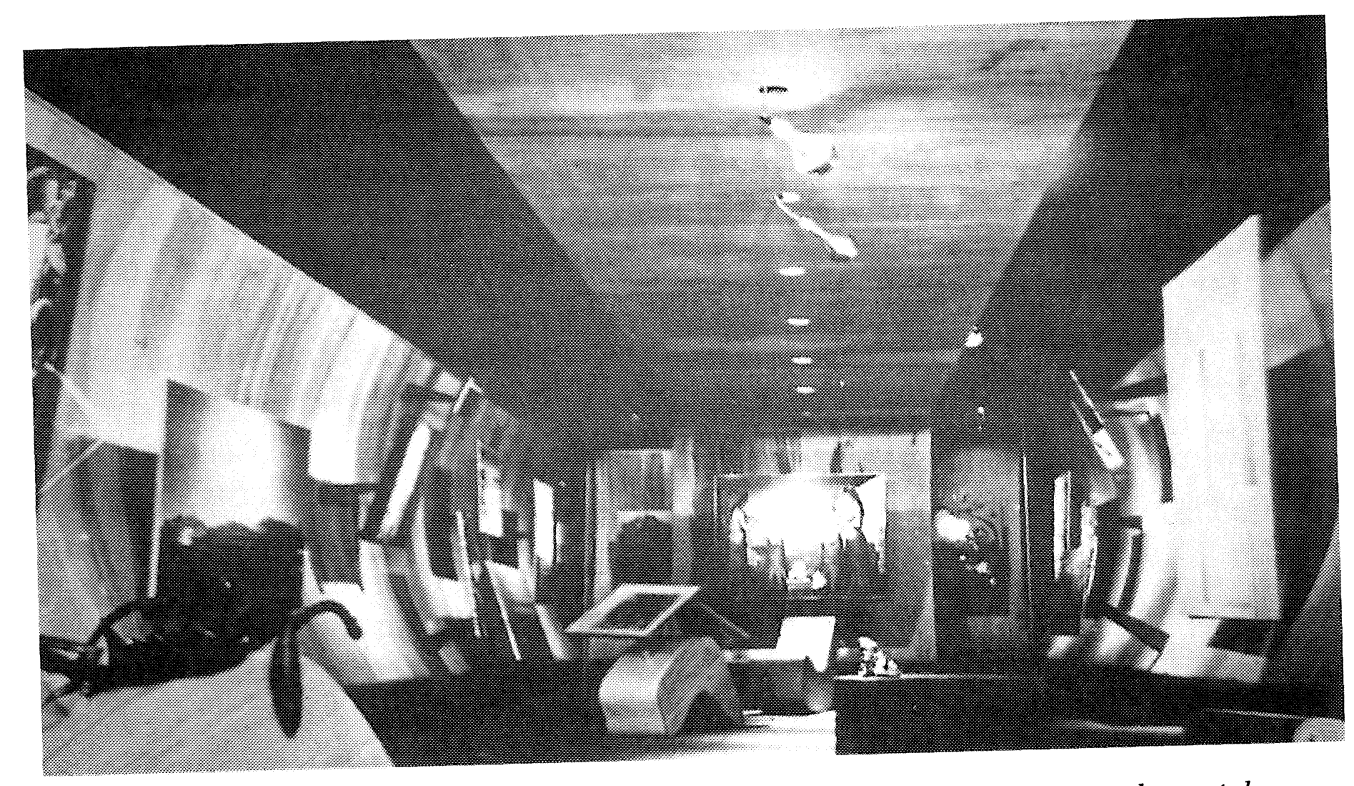

Galería de Peggy Guggenheim titulada: "The Art of this Century», donde los cuadros estaban colgados sobre bates de béisbol y apoyados en paredes curvas. 
Dos maneras de impulsar el arte: Peggy Guggenheim y...

Posteriormente, en 1943 en el llamado "Salón de Primavera», Peggy tuvo la visión futurista de abrir sus puertas a artistas desconocidos del momento, como Pollock, Motherwell, William Baziotes, Mark Rothko y Clifford Still, hoy reconocidos por todo el mundo como los grandes pilares de la Escuela de Nueva York. Muchas de las pinturas de estos últimos, fueron compradas por ella formando parte de su colección actual.

Hoy en Venecia, en el Palazzo Verner dei Leoni, bajo los ruidos de los turistas del Gran Canal, el ajetreo de las góndolas y los vaporetos puede verse la fantástica Colección de esta mujer, definida por Henry James «como un ser frío e impenetrable, capaz de estar en silencio y al mismo tiempo experta en escuchar y opinar».

Transformada en leyenda, Peggy allá donde se encuentre, dirigirá su mirada severa sobre un mundo más decadente del que ella misma vivió. Venecia se está hundiendo literalmente junto con su Palazzo, y, los hombres que pasaron por su vida: Laurence Vail ( (su primer marido), John Holms, Garman, y Max Ernst (su segundo marido), no consiguieron dejar huella en el alma de esta mujer, con corazón de águila real.

Cuando a los ochenta años, su amigo Gore Vidal, se acercó a felicitarle y le preguntó: ¿Cómo estás? ... Ella, enferma de un terrible problema vascular contestó: «Muy bien, para alguien que está a punto de morirse, no del todo mal»...

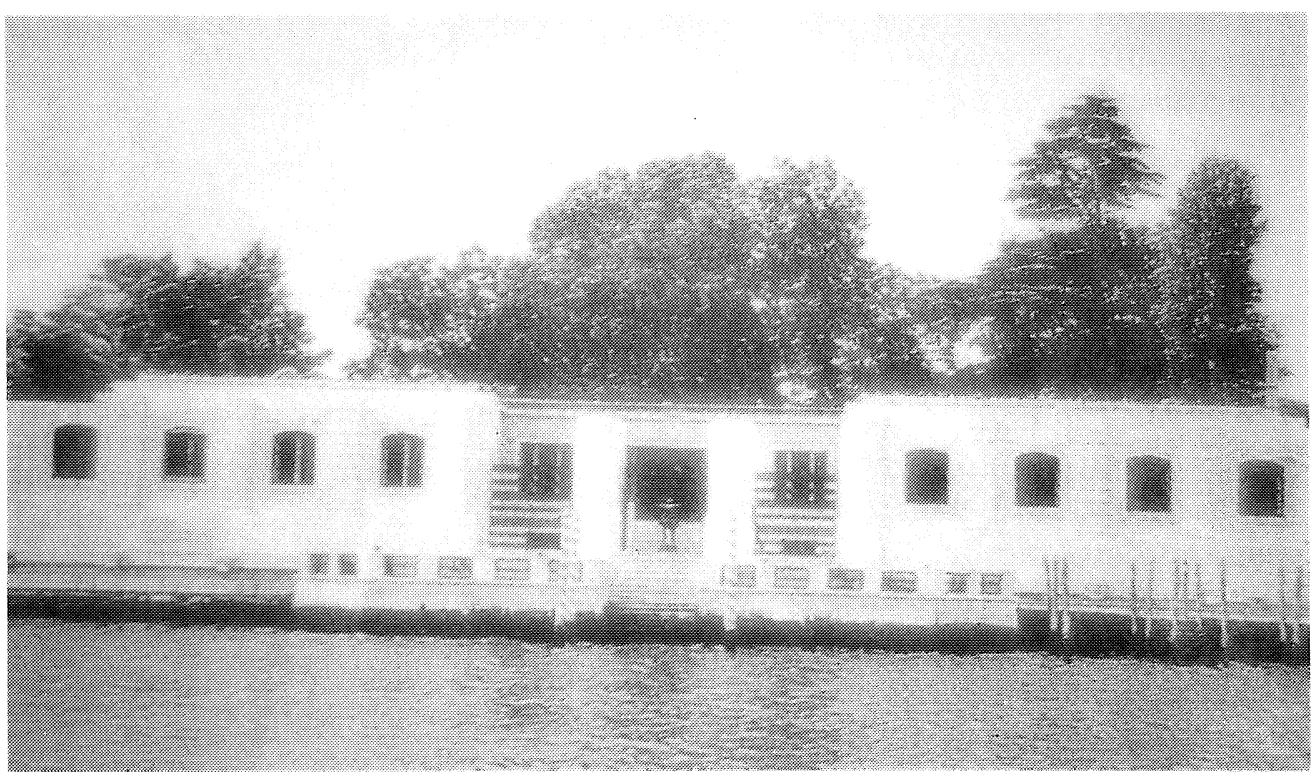

Palazzo Verner dei Leoni sobre el gran Canal de Venecia. 


\section{Gertrude Vanderbilt Whitney}

Otra mujer absolutamente decisiva en cuanto al desarrollo del arte del s.XX es Gertrude Vanderbilt , fundadora nada menos que del Whitney Museum de Nueva York, considerado hoy, como la Catedral del Arte Americano más vanguardista y valiente.

$\mathrm{Su}$ vida nada tiene que ver con la de Peggy Guggenheim.

Su visión de futuro, se remonta al año 1907, en el que organizó una exposición en el Club de Mujeres de Nueva York, del que era socia fundadora. En 1913, contribuyó con obras de su propiedad a la conocida exposición "Armory Show», y, durante muchos años subvencionó los déficits de la Sociedad de Artistas Independientes Americanos (fundada en 1914).

En ese año creó el «Whitney Studio» en el número 8 de West Eight Street, haciendo de él, una pequeña Galería que reunía periódicamente exposiciones de artistas americanos.

En 1818, creó el «Whitney Studio Club», al que podía acceder cualquier artista, siempre que estuviese presentado por un miembro del citado Club. De esta forma, los pintores tuvieron la oportunidad de mostrar su obra al público sin ser sometidos previamente a un jurado de censura. Pero Mrs. Whitney, no se contentó con esto, su ayuda a los creadores la llevó a cabo por medio de otros muchos procedimientos: enviando a los artistas a estudiar a Europa, pagando sus facturas médicas, los alquileres de sus estudios, y lo más importante, comprando sus obras.

En 1929 poseía más de 500 cuadros de artistas americanos y, su valentía y deci-

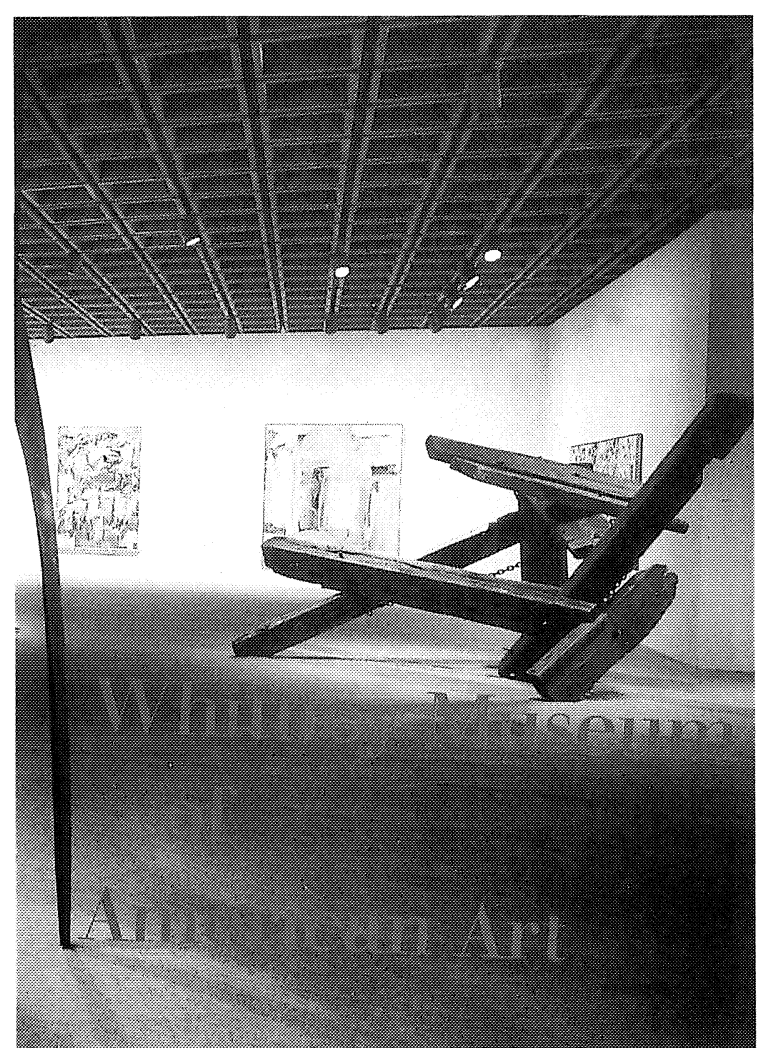

"Whitney Museum of American Art», creado por Gertrude Vanderbildt. 
Dos maneras de impulsar el arte: Peggy Guggenheim y...

sión le llevó a ofrecer este tesoro al Metropolitan Museum de Nueva York, cuyo Director (hombre conservador, timorato y necio), recusó el ofrecimiento...

Este contratiempo, como suele suceder normalmente a las mujeres luchadoras de gran carácter, le dio más fuerza de decisión y, así contestó al pacato Director anunciando públicamente que en Enero de 1930 abriría las puertas de su propio Museo, con un concepto radicalmente distinto de todos los anteriores: simplemente, el de ayudar a los artistas americanos. Efectivamente, este Museo, abrió sus puertas en Noviembre de 1931, con otra mujer como Directora, Juliana Force, que había sido la ayuda constante y fructífera de Mrs. Whitney.

Desde su nacimiento, el Museo ha cambiado dos veces de ubicación. En 1954, fué trasladado a un nuevo edificio en West Fifty-four Street, y, en 1966 a su sede actual, diseñada por Marcel Breuer y Hamilton Smith, con Michael Irving como arquitecto ayudante, en Madison Avenue 72 .

Para comprender un poco la filosofía de esta mujer, hay que entrar en su alma y oír sus palabras. He aquí su concepción sobre lo que iba a ser su gran empuje al Arte actual:

«Desde que se inventaron los Museos, los artistas liberales contemporáneos, tuvieron la gran dificultad y a veces la imposibilidad de cruzar sus puertas.

Los museos tienen la mala costumbre de esperar hasta que un pintor ó un escultor haya adquirido un cierto reconocimiento oficial, antes de aceptar su trabajo, dentro de sus sagrados muros.

Exactamente lo contrario es lo que se llevará a cabo en el Museo Whitney...»

Y así, obras iniciales de Arthur Dove, Marshden Hartley, Georgia O'Keeff, tuvieron entrada en el Museo para formar parte de su colección permanente.

Por cierto, las 500 obras, que formaban parte del núcleo inicial en 1930, hoy se han transformado en casi 10.000 . Mrs. Whitney hizo el esfuerzo de comprar 100 cuadros más, en 1931, para completar el Museo en el momento de su apertura, contando éste, pues en aquel instante crucial, con 600 cuadros.

Dos de los autores que recibieron mayor ayuda de esta inigualable mujer, fueron Eduard Hopper y Calder. La viuda del primero, donó en agradecimiento, del impulso de Mrs. Whitney a su marido, 2.500 obras del artista, constituyendo la mayor donación que un solo pintor haya hecho a ningún museo del mundo. 
La visión del arte vanguardista del s.xx de Gertrude Vanderbilt fue universal. En el $2^{\circ}$ piso de su Museo, donde se alberga su Colección Permanente, están presentes Pollock, Barnett Newman, Robert Raushenberg, Rothko y Clyfford Still, así como artistas posteriores, más controvertidos, por ejemplo Marshen Hartley, Donald Judd, de Kooning, Agnes Martin Reinhardt y David Smith, quienes tienen también su representación en la Colección del Museo.

El Realismo, el Expresionismo Abstracto (representado por la Escuela de Nueva York), el Surrealismo, la Nueva Abstracción, el Minimal Art, el Arte Cinético, el Op Art y el Pop Art, están allí recogidos.

Hoy, esta colosal Colección Permanente, se encuentra en el $2^{\circ}$ piso del Museo. El tercer piso, está dedicado al Minimal Art, a través de Agnes Martin. Y, a partir del $4^{\circ}$, se celebran exposiciones temporales de jóvenes artistas ignorados, (siguiendo las tesis de su fundadora), que probablemente, con el tiempo, después de este impulso por el templo neoyorkino del arte, llegarán a ser alguien.

Esta fue la voluntad, valentía y visión de futuro de una gran mujer, sin cuya existencia, muchos de estos autores serían unos perfectos desconocidos y cuya comprensión de la vida, me hace citar a Spencer, para discrepar frontalmente con su tesis, cuando dice: «Las emociones son las señoras, el intelecto el señor»...

Creo que ambos tiene que estar fusionados para llegar a ser algo, y en cualquier caso, en Mrs. Gertrude Vanderbilt Whitney las emociones, el intelecto, la capacidad, el auto-perfeccionamiento, la originalidad, la decisión, la entrega, la sabiduría y el trabajo tienen definitivamente alma y nombre de mujer...

\section{Bibliografía}

«Capollavori della Collezione Peggy Guggenheim».The Solomon R. Guggenheim Foundation. New York. 1994.

Guggenheim, PegGY. «Out of this Century» (Confessions of an Art Addict).Andre Deutsch. London. 1979.

SHORT, RoBERT. «Dada \& Surrelism».Laurence King. London. 1980.

"Whitney Museum of American Art». Whitney Museum. New York.1985.

PiERRE, Jose. «Le Surrealisme». De. Rencontre Laussanne.Paris. 1967.

Rocamora, Carmen. "Veinte museos de arte contemporáneo del mundo». De. CRG. Valencia. 1995. 